\title{
Atención obstétrica en adolescentes menores de 15 años
}

\author{
Alvaro Monterrosa*; Ana María Bello**
}

\begin{abstract}
RESUMEN: El embarazo en adolescentes es un problema con implicaciones psíquicas, sociales y médicas. En la Maternidad "Rafael Calvo", Cartagena, Colombia en el año de 1994 se atendieron 12.416 partos, siendo 3.161 pacientes (25.4\%) menores de 20 años de edad: estando en adolescencia temprana $57(1.8 \%$ ) y en adolescencia tardía $3.104(98.2 \%)$. Se comparan los resultados obstétricos de la atención a las adolescentes tempranas con los de 180 maternas adultas. La edad promedio de las adolescentes fue 13.9 años de edad. En ellas se presentó mayor frecuencia de: parto pretérmino y parto post-término, ruptura prematura de membranas, hipertensión inducida por el embarazo, hemorragia post-parto, contractilidad uterina alterada y retardo en el crecimiento intrauterino, aunque las diferencias no fueron estadísticamente significativas. El bajo peso al nacer fue más frecuente en adolescentes, y la diferencia si fue estadísticamente significativa, con un riesgo relativo 2 veces mayor. La presentación fetal, los desgarros perineales maternos, la talla y el apgar de los neonatos fue similar en ambos grupos. El embarazo en la adolescencia se debe prevenir, y ello se consigue con una correcta educación en salud reproductiva, incluida en un amplio y adecuado programa de educación sexual.
\end{abstract}

PALABRAS CLAVES: Adolescencia, embarazo en adolescentes, bajo peso al nacer, ruptura prematura de membranas, parto pretérmino, educación sexual, retardo en el crecimiento intrauterino.

SUMMARY: Pregnancy in teenager is a problem with psichical, social and medical implications. In the "Rafael Calvo" maternity, Cartagena, Colombia, 1944 were attended 12.416 birth deliveries, being 3.161 patients $(25.4 \%)$ younger than 20 years old: being in early adolescence $57(1.8 \%)$ and later adolescence $3.104(98.2 \%)$. Are compared the obstetrical results of the early teenagers attention withe the results of $\mathbf{1 8 0}$ maternal adults. The average age of teenagers was $\mathbf{1 3 . 9}$ years old. In them was presented a higher secuency of preterm birth and post term birth, early membranes breakdown, hipertensure induced by pregnancy, post part bleeding, altered uterine contractility and delay of uterine growth, although differences weren't statistically significatives.

Low weight at birth was more frecuent in teenagers with statistically significative difference, with a relative risk 2 times higher. The fetal presentation, maternal perineal breaks, sixe and Apgar of the neonates was simmilar in both groups. Pregnancy in the adolescence must be prevented, and that's gotten with a correct education in reproductive health, indiced in a wide and adequated sexual education program.

KEY WORDS: Adolescence, pregnancy in teenager, low weighy at birth, early membranes break, preterm birth, sexual education, delay of intrauterine growth.

\section{Introducción}

La organización mundial de la Salud estableció que la adolescencia transcurre entre los 10 y 19 años de edad (1), y es subdividida en adolescencia temprana: 10-14 años, y adolescencia tardía: 15-19 años (2). La adolescencia es la etapa del desarrollo que señala el final de la niñez y el inicio de la vida adulta. Desde un punto de vista biológico es un período de crecimiento y maduración, de aparición de los caracteres sexuales secundarios y de la adquisición de la capacidad reproductiva. A nivel psicológico se adquiere madurez en el desarrollo cognoscitivo, personalidad, valores ético-morales y el desarrollo de roles laborales de tipo adulto. A nivel social se produce la adaptación al grupo socio-cultural de su tiempo y entorno, es época de cambios, y en la medida en que estos coincidan o se aparten de los patrones considerados como normales, el adolescente se sentirá aceptado o rechazado por la sociedad (1-7).

\footnotetext{
* Docente. Departamento de Ginecología y Obstetricia. Facultad de Medicina. Universidad de Cartagena. Colombia.

** Estudiante. Departamento de Ginecología y Obstetricia. Facultad de Medicina. Universidad de Cartagena. Colombia.
}

Más de la mitad de la población mundial tiene menos de 25 años de edad, calculándose para 1994 la existencia de 1.582 millones de personas entre 10 y 24 años, y se prevé que la población mundial de adolescentes para el año 2.025 será de 2.044 millones (8-9).

El DANE según censo de población de 1994, establece que Colombia cuenta con 7'391.781 adolescentes (jóvenes entre 10-19 años de edad), existiendo un adolescente por cada 5 colombianos. 3'642.202 (49.2\%) de sexo femenino y $3^{\prime} 749.731(50.8 \%)$ de sexo masculino. $34 \%$ de los adolescentes residen en zonas rurales y el $66 \%$ en áreas urbanas. Se prevé que para el año 2.025 serán 11'200.000 (8-9).

Un número importante de adolescentes son sexualmente activos a edades tempranas y una proporción creciente de esta actividad sexual ocurre fuera del matrimonio. En los últimos 20 años se ha observado un aumento creciente de la frecuencia de embarazos en adolescentes (3,10-12). 15 millones de mujeres adolescentes paren cada año en el mundo, siendo una quinta parte de todos los nacimientos, y ocurriendo un buen porcentaje en países en desarrollo (9). 78 de cada mil adolescentes paren cada año en Colombia (13). En EEUU cada año se embaraza una de cada 10 adolescentes y el 
$12.7 \%$ de todos los nacimientos vivos de 1992 fueron de madres menores de 20 años de edad (2, 14-17). López Gómez (10) cita a Dickens y Allison, quienes consideraron al embarazo en la adolescencia como un fracaso de la sociedad, el hogar y la escuela.

El aumento en la frecuencia de los embarazos a tan temprana edad, cuando aún no existe la preparación física ni psíquica para afrontar las exigencias de la maternidad, es debida a varios factores:

1. Maduración sexual más temprana. La pubertad, con el inicio de los mecanismos endocrinos que permiten la capacidad reproductiva, suele presentarse hoy día a edades más tempranas que en generaciones previas. El establecimiento de este funcionamiento hormonal lleva implícito el despertar sexual. En Colombia la menarquia ocurre en promedio a los 12 años y la ovulación se regulariza en el año siguiente. $(6,12,18-23)$.

2. Inicio temprano de las relaciones sexuales. Eventos impulsados por la maduración sexual, pero sin duda influenciados por el medio ambiente rico en estímulos eróticos que inducen al coito, lo cual sumado a la mala fundamentación en educación sexual, permiten tener un joven no capacitado para tomar la decisión correcta $(6$, 15, 19-20, 24-25). Profamilia, El Instituto del Seguro Social y el Instituto Nacional de Cancerología realizaron en Colombia en 1993 la encuesta de conocimientos, actitudes y prácticas para determinar, entre otras variables, la sexualidad y comportamientos de riesgo para la salud en los adolescentes entre 12 y 17 años, encontrando que el $17 \%$ de los adolescentes habían tenido coitos, siendo mayor en varones $(26 \%)$ que entre mujeres $(8 \%)$. La edad promedio para la primera relación sexual fue 13.4 años en varones y 14.8 para las adolescentes, para un promedio de 13.7. Del total de adolescentes que habían tenido coitos, el $52 \%$ habían quedado en embarazo (8). El embarazo a edad temprana se traduce muy a menudo en altas tasas de fecundidad y riesgos de salud elevados para las madres y sus hijos $(8-9,19,24)$.

3. Ignorancia en planificación familiar. Los adolescentes carecen del conocimiento bien fundamentado de los diferentes métodos de planificación familiar. El método de planificación familiar ideal en la adolescencia es la abstinencia coital, pero si los coitos hacen parte de la vida sexual del adolescente, diversos métodos pueden ser utilizados de forma inocua y segura $(2,6,15,18-20,23,26)$.

4. Ignorancia en reproducción. Existe desconocimiento de la fisiología sexual y de los mecanismos de la reproducción humana $(3,11,23)$. En la encuesta de conocimientos, actitudes y prácticas (8), encontraron que el $21 \%$ de los adolescentes no consultan a nadie cuando tiene dudas sobre sexualidad. A mayor edad consultan más a los amigos, cuyas opiniones no deben ser las más apropiadas.

5. Genitalización de la sexualidad. Debido a la incorrecta educación sexual, se interpreta el coito como la única forma de dar y recibir placer. La sexualidad es una vivencia amplia, que involucra múltiples aspectos que son gratificantes y que no conllevan los riesgos de la maternidad precoz y las enfermedades de transmisión sexual. $(3,8-9)$.
A los anteriores se suma la falta de canales de comunicación dentro del entorno familiar, la pérdida del poder de manejo de la familia, y la necesidad de dependencia y protección (3, 27-30).

El embarazo en la adolescencia tiene implicaciones sociales negativas como: embarazo indeseado, madresolterismo, aborto provocado, hijo indeseado, abandono escolar, matrimonio forzado y en condiciones extremas intento de suicidio (2-3, 8, 12, 17-19). Muchos de los embarazos en la adolescencia son embarazos no deseados y suelen terminar como abortos provocados realizados en la clandestinidad al estar penalizado el aborto en Colombia (19). No se conoce la cuantificación real del embarazo en la adolescencia. Lo que podemos evaluar es la maternidad en la adolescencia, y se ha considerado la existencia de un mayor riesgo de resultados obstétricos adversos en este grupo etáreo, comparados con los de la población adulta $(3,11,16,18,25,29,31-32)$. Los efectos adversos se pueden magnificar si la adolescente es menor de 15 años $(6,10,22)$. El embarazo en la adolescencia conllevaría un mayor riesgo de toxemia, anemia, aborto espontáneo, parto pretérmino, infección urinaria, desnutrición e hijos con bajo peso al nacer (1012, 16, 18-19, 31-33).

Es nuestro objetivo comparar los resultados obstétricos, producto de la atención a pacientes en adolescencia temprana con los de población adulta, estableciendo diferencias, precisando su significancia estadística y estimando el riesgo relativo.

\section{Material y métodos}

Se revisaron las historias clínicas de todas las pacientes menores de 15 años de edad, que solicitaron atención obstétrica por estar en trabajo de parto con embarazo mayor de 28 semanas, al Hospital de Maternidad "Rafael Calvo", Cartagena, Colombia durante el año de 1994, las cuales conforman el grupo de estudio. Fueron escogidas al azar 180 historias clínicas de pacientes nulíparas, mayores de 19 años de edad, con feto único, mayor de 28 semanas de gestación que ingresaron en trabajo de parto al mismo centro y en el mismo año, conformando el grupo control.

Los resultados perinatales del grupo de estudio y del grupo control son comparados midiendo la significancia estadística de las diferencias (valor de P), utilizando la prueba de Mantel-Haenszel, y calculando el riesgo relativo. Los análisis estadísticos se realizaron con la ayuda del programa Epi-info 5.

\section{Resultados}

En 1994 en el Hospital de Maternidad "Rafael Calvo", Cartagena, Colombia se atendieron 12.416 partos, siendo $10.665(85.9 \%)$ vía vaginal y $1.751(14.1 \%)$ por operación cesárea.

3.161 pacientes $(25.4 \%)$ eran menores de 20 años de edad, estando en la adolescencia temprana $57(1.8 \%)$ de ellas y en la adolescencia tardía 3.104 (98.2\%).

Edad materna. Las 57 pacientes obstétricas en adolescencia temprana son evaluadas, teniendo edad promedio de $13.96+/$ - 0.19 años (Tabla 1 ) y comparadas con 
Tabla 1

\section{MATERNAS ADOLESCENTES (GRUPO ESTUDIO) DISTRIBUCION POR GRUPOS ETAREOS AÑOS DE EDAD}

\begin{tabular}{|lll|}
\hline Edad & No. & \% \\
\hline 13 & 2 & 3.5 \\
14 & 55 & 96.5 \\
Total & 57 & 100. \\
\hline
\end{tabular}

Monterrosa-Bello.

$X^{=}=13.96 \pm 0.19$

Tabla 2

\section{MATERNAS ADULTAS (GRUPO CONTROL) DISTRIBUCION POR GRUPOS ETAREOS} AÑOS DE EDAD

\begin{tabular}{|lll|}
\hline Edad & No. & \% \\
\hline $20-22$ & 101 & 56.1 \\
$23-25$ & 68 & 37.8 \\
$26-28$ & 11 & 6.1 \\
Total & 180 & 100. \\
\hline
\end{tabular}

Monterrosa-Bello.

$X^{=}=22.5 \pm 1.84$

180 maternas nulíparas adultas con edad promedio de 2.3 +/- 1.84 años (Tabla 2).

Paridad. 53 (92.9\%) de las adolescentes cursaban su primera gestación y $4(7.1 \%)$ tenían antecedente de un aborto. Entre las adultas 164 (91.1\%) cursaban su primera gestación y $16(8.9 \%)$ habían tenido un aborto previo.

Edad gestacional. El $82.4 \%$ (47) de las adolescentes y el $88.9 \%$ (160) de las adultas tuvieron parto de término. $\mathrm{X}^{2}=1.61 \mathrm{P}=0.2$ RR: 0.93. El 10.6\% (6) de las adolescentes y el $7.2 \%$ (13) de las adultas tuvieron parto pretérmino. $\mathrm{X}^{2}=0.6 \mathrm{P}=0.4 \mathrm{RR}$ : 1.46 . El 7.0\% de las adolescentes y el $3.8 \%$ de las adultas parieron en período post término $\mathrm{X}^{2}=0.9 \mathrm{P}=0.3 \mathrm{RR}: 1.80$. (Tabla 3).

Presentación fetal. No se presentaron embarazos múltiples en las adolescentes, $56(98.2 \%)$ tenían feto cefálico y $1(1.8 \%)$ feto podálico. En las adultas 174 $(96.7 \%)$ tenían feto cefálico y $6(3.3 \%)$ podálico. $\mathrm{X}^{2}=0.3$ $\mathrm{P}=0.5 \mathrm{RR}: 1.02$.

Vía del parto. El $89.5 \%$ (51) de las adolescentes y el $85.5 \%$ (154) de las adultas tuvieron parto vaginal. Cesárea el $10.5 \%$ (6) de las adolescentes y el $14.5 \%$ (26) de las adultas. $\mathrm{X}^{2}=0.57 \mathrm{P}=0.4 \mathrm{RR}$ : 1.05 . Desproporción cefalopélvica fue el motivo por el cual se realizó cesárea a las 6 adolescentes $(10.5 \%)$. La misma causa 1 obligó a realizar cesárea a 16 de las pacientes adultas $(8.9 \%) \mathrm{X}^{2}=$
Tabla 3

\section{DISTRIBUCION SEGUN EDAD GESTACIONAL SEMANAS DE GESTACION GRUPO DE ESTUDIO Y GRUPO CONTROL}

\begin{tabular}{|ll|ll|ll|}
\hline Edad & & \multicolumn{2}{|c|}{ Adolescentes } & \multicolumn{2}{c|}{ Adultas } \\
Gestacional & & No. & \% & No. & \% \\
\hline-32 & $*$ & 1 & 1.8 & 5 & 2.8 \\
$33-36$ & $*$ & 5 & 8.8 & 8 & 4.4 \\
$37-42$ & $* *$ & 47 & 82.4 & 160 & 88.9 \\
+43 & $* * *$ & 4 & 7.0 & 7 & 3.8 \\
Total & & 57 & 100 & 180 & 100 \\
\hline
\end{tabular}

Monterrosa-Bello.

$$
\begin{array}{ll}
* & \mathrm{X}^{2}=0.6 \mathrm{P}=0.4 \text { RR: } 1.46 \\
* * & \mathrm{X}^{2}=1.61 \mathrm{P}=0.2 \mathrm{RR}: 0.93 \\
* * * & \mathrm{X}^{2}=0.9 \mathrm{P}=0.3 \mathrm{RR}: 1.80
\end{array}
$$

$0.14 \mathrm{P}=0.7 \mathrm{RR}: 1.18$ (Tabla 4). Todas las presentaciones podálicas en las pacientes adultas fueron atendidas por cesárea. En el caso de la paciente adolescente, dificultades institucionales impidieron realizar cesárea, siendo atendido por vía vaginal.

Complicaciones maternas. 6 pacientes adolescentes $(10.5 \%)$ y 15 adultas (8.3\%) presentaron hipertensión inducida por la gestación. $\mathrm{X}^{2}=0.26 \mathrm{P}=0.6 \mathrm{RR}$ : 1.26. Dos pacientes adolescentes (3.5\%) y cinco adultas (2.8\%) fueron inducidas por estar de post-término sin inicio en el trabajo de parto $\mathrm{X}^{2}=0.88 \mathrm{P}=0.7 \mathrm{RR}$ : 1.26. Siete pacientes adolescentes $(12.3 \%)$ y 17 adultas $(9.4 \%)$ presentaron alteraciones en la dinámica de la contractilidad uterina, ameritándose conducción ocitocica. $\mathrm{X}^{2}=0.3 \mathrm{P}=$ $0.5 \mathrm{RR}: 1.30$. De éstas, todas las adolescentes alcanzaron el parto vaginal y 2 adultas ameritaron cesárea. Ruptura prematura de membranas presentaron 8 de las adolescentes $(14 \%)$ y 12 de las adultas $(6.6 \%) \mathrm{X}^{2}=3.03 \mathrm{P}=0.08 \mathrm{RR}$ : 2.1. Retardo en el crecimiento intrauterino presentaron 2 adolescentes $(3.5 \%)$ y una adulta $(0.5 \%) . \mathrm{X}^{2}=3.01 \mathrm{P}=$ 0.08 RR: 6.32 . Una adolescente $(1.7 \%)$ y una adulta $(0.5 \%)$ presentaron hemorragia post parto $\mathrm{X}^{2}=0.74 \mathrm{P}=$ 0.38 RR: 3.16. Desgarros perineales presentaron 4 adolescentes $(7.0 \%)$ y 14 adultas $(7.7 \%) \mathrm{X}^{2}=0.04 \mathrm{P}=0.8 \mathrm{RR}$ : 0.9. No se presentaron otras complicaciones quirúrgicas o médicas. No se presentaron muertes maternas.

Valoración neonatal. En ambos grupos todos los fetos nacieron vivos. De los 6 recién nacidos prematuros, hijos de adolescentes, 1 falleció por insuficiencia respiratoria mientras de los 13 recién nacidos, hijos de maternas adultas, 2 fallecieron por prematurez. 11 recién nacidos $(19.2 \%)$ de adolescentes y $17(9.5 \%)$ de adultas tuvieron bajo peso al nacer, menos de 2.500 gramos. $X^{2}$ $=4.02 \mathrm{P}<0.05$ RR: 2.04 . Siete $(12.3 \%)$ de los hijos de adolescentes y $22(12.2 \%)$ de las adultas pesaron entre 3.501 y 4.000 gramos. Tuvieron más de 3.000 gramos de peso al nacer el $47.4 \%$ (27) de los nacidos de madres adolescentes y el $61.1 \%$ (110) de las adultas $\mathrm{X}^{2}=3.34 \mathrm{P}=$ $0.06 \mathrm{RR}: 0.78$. El peso promedio de los recién nacidos de 
Tabla 4

DISTRIBUCION SEGUN VIA DEL PARTO
MOTIVACION DE LA CESAREA
GRUPO DE ESTUDIO Y GRUPO CONTROL

\begin{tabular}{|l|ll|ll|}
\hline Vía & \multicolumn{3}{|c|}{ Adolescentes } & \multicolumn{2}{c|}{ Adultas } \\
del parto & No. & \% & No. & \% \\
\hline No. Cesárea (Vaginal)* & 51 & 89.5 & 154 & 85.5 \\
Cesárea: (D.C.P.1)** & 6 & 10.5 & 16 & 8.9 \\
Cesárea: (podálica) & $=$ & $=$ & 6 & 3.4 \\
Cesárea: (S. Fetal) & $=$ & $=$ & 2 & 1.1 \\
Cesárea: (D. dinámica) & $=$ & $=$ & 2 & 1.1 \\
Total Población & 57 & 100 & 180 & 100 \\
\hline
\end{tabular}

Monterrosa-Bello.

$\begin{array}{ll}1 & \text { D.C.P.: Desproporción céfalo-pélvica. } \\ * & \mathrm{X}^{2}=0.57 \mathrm{P}=0.4 \mathrm{RR}: 1.05 \\ * * & \mathrm{X}^{2}=0.14 \mathrm{P}=0.7 \mathrm{RR}: 1.18\end{array}$

Tabla 5

DISTRIBUCION DE LOS NEONATOS

SEGUN PESO AL NACER. GRUPO DE ESTUDIO Y GRUPO CONTROL

\begin{tabular}{|l|ll|lr|}
\hline \multirow{2}{*}{ Peso Al nacer } & \multicolumn{2}{|c|}{ Adolescentes* } & \multicolumn{2}{c|}{ Adultas** } \\
(Gramos) & No. & \% & No. & \% \\
\hline $1.501-2.000 *$ & 1 & 1.7 & 3 & 1.7 \\
$2.001-2.500$ & 10 & 17.5 & 14 & 7.8 \\
$2.501-3.000$ & 19 & 33.3 & 53 & 29.4 \\
$3.001-3.500$ & 20 & 35.1 & 88 & 48.9 \\
$3.501-4.000$ & 7 & 12.3 & 22 & 12.2 \\
Total & 57 & 100 & 180 & 100 \\
\hline
\end{tabular}

Monterrosa-Bello.

$\begin{array}{ll}* & \mathrm{X}=2.943,1 \pm 484 \\ * * & \mathrm{X}=3.061,6 \pm 428 \\ * & \mathrm{X}^{2}=4.02 \mathrm{P}<0.05 \text { RR: } 2.04\end{array}$

madres adolescentes fue $2.943+/-484$ gramos y de los hijos de adultas $3.061+/-428$ gramos (Tabla 5). El 78.9\% (45) de los hijos de adolescentes y el 75\% (135) de los hijos de adultas tuvieron talla superior a 47 centímetros $\mathrm{X}^{2}=0.37 \mathrm{P}=0.5 \mathrm{RR}$ : 1.05. La talla promedio de los hijos de adolescentes fue $48.1+/-2.5$ centímetros y de los hijos de adultas fue $47.5+/-2.8$ centímetros (Tabla 6). Los hijos de las adolescentes tuvieron apgar mayor de 8 al minuto el $86.0 \%$ (49) y al quinto minuto el $91.3 \%$ (52). Los hijos de las adultas tuvieron apgar mayor de 8 al minuto el $85.5 \%$ (154) y al quinto minuto el $91.6 \%$ (164). Seis $(10.5 \%)$ de los hijos de adolescentes y $19(10.5 \%)$ de los hijos de adultas nacieron meconiados. $5(8.8 \%)$ de los hijos de adolescentes y $11(6.1 \%)$ de los de adultas, presentaron sepsis neonatal. $\mathrm{X}^{2}=0.48 \mathrm{P}=0.4 \mathrm{RR}: 1.49$. Falleció 1 (1.7\%) de los hijos de madres adolescentes, producto de embarazo de 28 semanas, en podálica, con
Tabla 6

\section{DISTRIBUCION DE LOS NEONATOS SEGUN TALLA AL NACER. GRUPO DE ESTUDIO Y GRUPO CONTROL}

\begin{tabular}{|c|c|c|c|c|}
\hline $\begin{array}{l}\text { Talla } \\
\text { Al nacer }(\mathrm{cms})\end{array}$ & \multicolumn{2}{|c|}{ Adolescentes* } & \multicolumn{2}{|c|}{ Adultas** } \\
\hline $38-40$ & 1 & 1.7 & 5 & 2.8 \\
\hline $41-43$ & 1 & 1.7 & 9 & 5.0 \\
\hline $44-46$ & 10 & 17.5 & 31 & 17.2 \\
\hline $47-49$ & 28 & 49.1 & 97 & 53.9 \\
\hline $50-52$ & 17 & 29.8 & 38 & 21.1 \\
\hline Total & 57 & 100 & 180 & 100 \\
\hline
\end{tabular}

Monterrosa-Bello.

$$
\begin{array}{ll}
* & \mathrm{X}^{=}=48.1 \pm 2.5 \\
* * & \mathrm{X}^{=}=47.5 \pm 2.8 \\
* & \mathrm{X}^{2}=0.37 \mathrm{P}=0.5 \text { RR: } 1.05
\end{array}
$$

ruptura prematura de membranas. Presentó síndrome de dificultad respiratoria secundario a membrana hialina, sepsis neonatal y hemorragia intraventricular. $4(2.2 \%)$ de los hijos de las pacientes adultas, fallecieron: dos por prematuridad, uno con historia de ruptura prematura de membranas, trabajo de parto prolongado, sufrimiento fetal, nacido meconiado y sepsis neonatal. El otro con trabajo de parto prolongado y posterior cesárea por desproporción céfalo-pélvica, nacido severamente deprimido.

\section{Discusión}

3.161 adolescentes tuvieron parto en el Hospital de Maternidad "Rafael Calvo" de Cartagena, Colombia en 1994 , siendo el $25.4 \%$ de toda la población, o sea un parto en adolescente por cada 4 de adultas. Cifra que es muy superior a la señalada en otros estudios $(3,19,32-34)$ y similar a la presentada por Moreno (6). A esta institución 
acuden pacientes de bajo nivel socioeconómico y cultural, en quienes pueden influenciar más los factores que ocasionan el embarazo a tan temprana edad $(3,7,32) .57$ pacientes en adolescencia temprana se convirtieron en madres, siendo el $1.8 \%$ de las adolescentes y el $0.4 \%$ de toda la población, o sea un parto en adolescente menor de 15 años por cada 162 maternas adultas.

El promedio de la edad en las maternas en adolescencia temprana fue de 13.9 años. Si la menarquia en promedio para la población colombiana actual es de 12 años y la ovulación suele regularizarse en el año siguiente $(7-8,12$, 19), estas "niñas" se están embarazando inmediatamente establecida la pubertad, ya que están teniendo coitos precoces, sin utilizar métodos contraceptivos (23). Aunque no fue evaluado en la presente serie, muchos de los embarazos a edades tan tempranas suelen ser indeseados, como lo señala el estudio de Germán López (35) del Centro para Jóvenes de Profamilia, Bogotá, en el cual tomaron en consideración 861 jóvenes que en 1991 acudieron a dicho centro solicitando le realizaran prueba de embarazo por tener episodio de atraso menstrual. El $40 \%$ de las adolescentes eran menores de 16 años, el 67\% eran estudiantes de secundaria, el $80 \%$ no usaban métodos contraceptivos. El 53\% tuvo prueba de embarazo positiva y el $8 \%$ de éstas últimas manifestó no desear el embarazo. Uriza (35) en 1983 comentó: "El embarazo en este período de la vida va a ser en su gran mayoría indeseado, va a provocar tensiones psicológicas, desajustes familiares profundos, complicados con la falta de apoyo familiar o social y la falta de preparación del personal de salud responsable de atender a la madre adolescente".

4 de las 57 adolescentes maternas a tan temprana edad, tenían antecedente de otra gestación que había terminado en aborto. La reincidencia en el embarazo es debido muy probablemente a fallas en la administración de anticonceptivos post aborto y a la falta de políticas educativas en salud reproductiva $(19,23)$. Es alta la reincidencia del embarazo en la adolescencia (18).

En nuestra serie no se investigó sobre el control prenatal de la gestación. Pardo, Uriza (19) en un estudio que agrupó 11 instituciones colombianas de II y III nivel, evaluaron 3.364 adolescentes $(6.17 \%)$ de 54.497 partos y encontraron que el $58.2 \%$ de las jóvenes no tuvieron control prenatal. Del resto el $25.7 \%$ tuvieron uno o dos controles, $63.9 \%$ tuvo tres a seis controles y más de seis controles sólo el $10.4 \%$. Esparza y Calle (33) encontraron una mayor proporción de ausencia de control prenatal en adolescentes comparadas con adultas. Igual aseveran Fraser et al (16). Existe una relación inversamente proporcional entre control prenatal y morbi-mortalidad materna y perinatal. Pardo y Uriza (19) encontraron que las complicaciones del parto y del puerperio fueron más frecuentes entre adolescentes sin control prenatal.

La mayoría de las pacientes, $82.4 \%$ de las adolescentes y el $88.9 \%$ de las adultas, tuvieron parto de término, siendo el porcentaje de éstas últimas superior, aunque sin diferencia estadística significativa. El parto pretérmino se dio con más frecuencia en adolescentes, sin diferencia estadística significativa, pero con un riesgo relativo de 1.46. Monterrosa (36), evaluando en el mismo centro los factores de riesgo al parto pretérmino encontró para menores de 19 años un RR: cifra muy superior a la determinada en esta evaluación, donde sólo se consideran menores de 15 años de edad. Es controversial si la adolescencia per se, es factor de riesgo al parto pretérmino ya que éste puede ser modificado por muy diversos factores, incluido el stress. La adolescente puede que esté agobiada por la incertidumbre o con temores sobre su integridad al momento del parto. La inmadurez biológica y la deficiente adaptación uterina han sido considerados $(12,36)$.

Encontramos una mayor frecuencia de embarazos de post-término en adolescentes tempranas que entre las adultas controles, sin diferencia estadística significativa pero con un riesgo relativo del doble. La inmadurez biológica podría llevar al no establecimiento de los mecanismos inmuno-endocrinológicos y psíquicos que tienen que ver con la preparación y el desencadenamiento del trabajo de parto.

No se encontraron diferencias con respecto a la presentación fetal. La única presentación anómala fue la presentación podálica. Las causales de presentación podálica son: estrechez pélvica, tumoración o malformación uterina, gran paridad, placenta previa, oligoamnios, polihidramnios, brevedad de cordón, macrosomía fetal, hidrocefalia, prematurez. La adolescencia no se considera factor predisponente para presentación podálica (3739). Más del $96 \%$ de los fetos de ambos grupos estaban en presentación cefálica.

El parto vaginal se presentó con más frecuencia en adolescentes $(89.5 \%)$ que en adultas $(85.5 \%)$, por tanto en este último grupo fue más frecuente la cesárea (14.5\%) mientras que en adolescentes fue el $10.55 \%$. Varios autores $(3,12,18-19,33)$ han aseverado que la adolescencia no es un factor indicativo para cesárea, como hemos demostrado. En toda paciente se requiere una juiciosa y adecuada valoración obstétrica para establecer la relación pelvis presentación fetal. Las normas obstétricas y no la edad de la paciente, son los indicadores de la conducta que se deben seguir en la atención del parto (6). A 6 de las pacientes adolescentes $(10.5 \%)$ y $16(8.9 \%)$ de las adultas, se les realizó cesárea por desproporción céfalo-pélvica, diferencia que no es estadísticamente significativa y con un RR de sólo: 1.18, cifras bastante bajas que nos permite aseverar que en las adolescentes, aún en menores de 15 años, no es obligatorio realizar cesárea. Posiblemente inmediatamente se da la pubertad se presentan modificaciones en la cintura pélvica, con una preparación temprana para permitir una gestación y un parto precoz. La pelvis de la adolescente suele tener características parecidas a la de la mujer adulta (12).

Con un riesgo relativo de aproximadamente 1.3 la adolescencia facilita el embarazo de post-término y las alteraciones en la dinámica uterina, lo cual se puede deber a inmadurez neuro-endocrina o inmunológica, pero con buena capacidad de respuesta al estímulo de los ocitócicos parenterales. Fue satisfactoria la respuesta de todas las adolescentes a los ocitócicos, con el establecimiento o regulación de la contractilidad uterina hasta llegar al parto normal. 
El riesgo de ruptura prematura de membranas en la adolescencia temprana fue 2 veces mayor con respecto a la población adulta, no siendo la diferencia significativamente estadística, pero sí clínicamente importante. La ruptura prematura de membranas fue la primera morbilidad asociada al embarazo en la adolescencia $(28.4 \%)$ encontrada en el estudio de las 11 instituciones colombianas (19). Son numerosos los factores predisponentes de ruptura prematura de membranas y son importantes las implicaciones obstétricas, entre ellas se destacan: aumento en el riesgo de parto pretérmino, bajo peso al nacer, infección perinatal, sepsis neonatal, anoxia, síndrome de dificultad respiratoria, prolapso de cordón y parto traumático. En la madre: amnionitis, e infección post parto o endometritis hasta infección pélvica (38-39).

El rechazo a la gestación o la gestación oculta del entorno familiar, puede llevar a ausencia o a insuficiente control prenatal, con la predisposición a anemia, desnutrición o hipovitaminosis (22). Todo lo anterior y el stress, la ausencia del reposo físico-psíquico adecuado, pueden llevar directamente o por la aparición de patologías diversas al retardo en el crecimiento intrauterino (22). Encontramos un riesgo relativo 6 veces mayor para retardo en el crecimiento intrauterino en pacientes en adolescencia temprana comparadas con adultas. Esta condición favorece alteraciones funcionales y adaptativas en el neonato (40). Además, los embarazos a temprana edad, repetidos a breves intervalos, agravan la carencia nutricional de la adolescente, aumentando las posibilidades de complicaciones maternas y perinatales, asevera Duarte Contreras (22). Los mismos factores sociales y médicos se consideran que están involucrados en el establecimiento de la hipertensión inducida por el embarazo (22), entidad de causa no conocida, explicada en parte por ausencia de adaptación a cambios endoteliales (38). La hipertensión inducida por el embarazo tiene elevadas tasas de morbilidad y mortalidad materna y perinatal (19). Monterrosa (41) en 1989 evaluó en el Hospital de Maternidad "Rafael Calvo" 9.939 partos, 51 complicados con eclampsia, siendo 19 (37.2\%) adolescentes entre 13 y 18 años y estableciendo un riesgo relativo de tener eclampsia en la adolescencia con respecto a la edad adulta de 3.7. En la presente serie el $10.5 \%$ de las adolescentes menores de 15 años presentaron hipertensión inducida por el embarazo, pero ninguna tuvo eclampsia. La diferencia no es estadísticamente significativa, con respecto al grupo control, pero la entidad fue más frecuente en adolescentes. Duarte (22) comenta que algunos autores encuentran una frecuencia cinco veces mayor de hipertensión inducida por el embarazo en adolescentes que en población adulta. Pardo y Uriza (19) encontraron preeclampsia en el $25.6 \%$ de las maternas adolescentes de 15 años. La frecuencia de preeclampsia se aumenta ante la falta de control prenatal (10).

Se presentó mayor frecuencia de hemorragia post parto en las adolescentes, en una relación de 3:1 con respecto a las adultas, sin diferencia estadística significativa. Suponemos falla en los mecanismos endocrinológicos de la contractilidad uterina, que podrían explicar este aumento en la frecuencia de la patología hemorrágica post-parto, debida sobre todo a atonía uterina. Igual encontraron Esparza y Calle (33).

Desde un punto de vista global encontramos que las complicaciones maternas fueron más frecuentes en adolescentes que en adultas, aunque no se observaron diferencias con significancia estadística, excepto en la presentación de desgarros perineales, donde los porcentajes fueron similares. La adolescencia no es factor de riesgo para desgarros perineales, éstos guardan relación con la calidad en la atención obstétrica. Existe controversia sobre los riesgos de complicaciones maternas en la adolescente embarazada (3). Algunos autores $(18,31,42)$ afirman que el embarazo en la adolescencia es una condición de muy alto riesgo. Otros señalan que sí existe riesgo obstétrico en el embarazo en la adolescencia, pero que es superable con un adecuado control prenatal $(2-3,18-19,25,29,32)$. Otros determinan que sólo hay aumento en la morbilidad entre adolescentes muy jóvenes, al no estar completa la madurez somática y emocional $(6-7,10,22)$.

El 19.2\% de los hijos de adolescentes y el $9.5 \%$ de las adultas tuvieron bajo peso al nacer, menos de 2.500 gramos de peso, siendo la diferencia estadísticamente significativa y definiendo un riesgo relativo de 2 . Estos hallazgos corresponden con los reportados por Khwaja et al (43) y con lo informado por López Gómez (10) en Venezuela. Este es uno de los puntos de vista donde hay más uniformidad entre los autores (40), siendo varios los factores que explicarían el bajo peso al nacer. Aunque Moreno (6) basado en varias revisiones y resultados propios, considera que el bajo peso al nacer, la prematurez y la adolescencia no tienen la importancia que se les ha pretendido dar y que las cifras se pueden mejorar con un adecuado control prenatal. Slap y Schawrs (44) correlacionaron el bajo peso al nacer con varios factores como: pocas visitas prenatales, historia del primer niño con bajo peso al nacer, trauma durante el embarazo, baja talla corporal materna, deficiencias nutricionales y otros factores que son comunes en las madres adolescentes como: bajo nivel socioeconómico y uso de alcohol, cigarrillo o drogas.

No se observaron diferencias entre los dos grupos con respecto a la talla y a la puntuación del apgar. Se presentó una mayor frecuencia de sepsis neonatal en hijos de madres adolescentes, debido tal vez a la mayor frecuencia de ruptura prematura de membranas, pero la diferencia no es significativa. Las enfermedades de transmisión sexual adquiridas en el embarazo pueden producir infección en el feto y en el neonato (14).

El embarazo en la adolescencia debe ser manejado por un equipo interdisciplinario, para brindarle una buena atención obstétrica, psicológica, social y educativa, fomentando una conducta más responsable y madura, para que la adolescente que se ha convertido en madre tome una actitud positiva frente a su nueva condición de vida $(3,7,22,31)$.

Existe evidencia indiscutible del aumento del embarazo en las adolescentes y en edades cada vez más tempranas, con el elevado costo social que ello conlleva tanto para la madre como para el recién nacido $(8-9,15$, 17). Si bien con el control prenatal podemos minimizar 
los efectos médicos deletéreos $(19,22)$, que persisten las implicaciones sociales que incrementan la pobreza y la baja calificación laboral de estas madres $(3,6,45)$. Por tanto se amerita una atención especial por parte de las organizaciones encargadas de la atención en salud, para prevenir el embarazo en la adolescencia. Prevención que sólo se conseguirá con una correcta educación en salud reproductiva, incluida en un amplio y adecuado programa de educación sexual $(6,10,19,22,45-47)$.

\section{Agradecimientos}

Al Profesor Armando Morales, de la U. de Cartagena, por la Asesoría Estadística. Al estudiante Néstor Llinás, por la colaboración en la recolección de datos. A Josefina Castellar, de la Biblioteca Ciencias de la Salud de la U. de Cartagena y a Clemencia Leyva de la Biblioteca Profamilia, Bogotá, por la colaboración en la búsqueda bibliográfica.

\section{BIBLIOGRAFIA}

1. O.M.S./F.N.U.A.P./U.N.I.C.E.F.: Higiene de la reproducción en la adolescencia. Estrategia de acción. 1989.

2. Laverde E., Contreras L., Rodríguez E. Embarazo en adolescentes: repercusiones psíquicas. Actualizaciones Pediátricas. 1993; 3(3): 116-125.

3. Barrios J., Ramos A. Adolescencia y embarazo. Aspectos perinatales y socioeconómicos. H.M.R.C. Cartagena, Colombia. Rev. Col. Obstet. Ginecol. 1993; 44(2): 101-106.

4. Klein R. Programas de intervención para el adolescente. En: La psicología en el ámbito perinatal. INPER. México 1988; 488.

5. Tolber K. La adolescente embarazada: características y riesgos psicosociales. En: La psicología en el ámbito perinatal. INPER. México. 1988.

6. Moreno A. Adolescentes y embarazo. Rev. Col. Obstet. Ginecol. 1989; 40(3): 227-232.

7. Berdugo G., Ballesteros G., Morales A. Adolescencia, embarazo y trabajo. Temas de enfermería. 1993; 20: 1-26.

8. Ordóñez M., Ojeda G. Adolescentes: sexualidad y comportamientos de riesgo para la salud, según encuesta de conocimientos, actitudes y prácticas. Tomo IV. I.N.C., I.S.S., Profamilia. Fondo de promoción y desarrollo de la salud. Bogotá. 1994.

9. Population Reference Bureau. The center for Population Options. La juventud mundial de 1994: Un enfoque especial sobre su salud reproductiva. Marzo 1994.

10. López-Gómez R. Adolescente embarazada. Rev. Obstet. Ginecol. Venezuela 1992; 52(1): 17-22.

11. Barrios J., Ramos A., Arévalo J et al. Aspectos perinatales de las adolescentes. Rev. Col. Obstet. Ginecol. 1985; 36: 102-112.

12. Duarte A. Embarazo en adolescentes. En: Ginecología de la niña y de la adolescente. 1a. Edición. Salvat. Bogotá. 1988; 443-461.

13. Prada E., Singh S., Wulf D. Adolescentes de hoy, padres del mañana. 1a Edición. Inst. Alan Guttmacher. Bogotá. 1989.

14. Hoekelman R. Teenager pregnancy. One of our nation's most challenging dilemmas. Pediatrics Annals. 1993; 22(2): 81-82.

15. Alexander $\mathrm{CH}$., Guyer B. Adolescent pregnancy: occurrence and consequences. Pediatric Annals. 1993; 22(2): 85-88.

16. Fraser AM., Brockert JE., Ward RH. Association of young maternal age with adverse reproductive outcomes. The New Engl. J. Med. 1995; 332(17): 1113-1117.

17. Goldemberg RL., Klerman LV. Adolescent pregnancy another look. The New Engl. J. Med. 1995; 332(17): 1161-1162.

18. Sánchez P., Mendieta N. Embarazo en la adolescencia. Experiencia de la A.S.C.P. Rev. Col. Obstet. Ginecol. 1994; 45(3): 208-212.

19. Pardo F., Uriza G. Estudio de embarazo en adolescentes en 11 instituciones colombianas. Rev. Col. Obstet. Ginecol. 1991; 42(2): 109-121.

20. Elster A., Panzarine S., McAnarney E. Causes of adolescent pregnancy. Medical aspect of human sexuality 1980; 14(7): 22-28.

21. Duarte A., Barreto L. Factores determinantes del embarazo en adolescentes solteras. Rev. Col. Obstet. Ginecol. 1985; 36(3): 291-306.

22. Duarte A. Manejo de la adolescente embarazada. Rev. Col. Obstet. Ginecol. 1990; 41(1): 25-30.

23. López G. Anticoncepción en adolescentes. En: $2^{\circ}$ Seminario Colombiano de Sexualidad en Adolescentes. (Memorias): 1993; 77-82.

24. Flick LH. Paths to adolescent parenthood: Implications for prevention. Public Health Rep. 1986; 101(2): 132-147.
25. Arechavaleta H., Uzcátegui O. Embarazo en adolescentes. Rev. Obstet. Ginecol. Venezuela. 1985; 45(2): 38-42.

26. Zelnick M., Kantner JF. Sexual activity, contraceptive use and pregnancy among metropolitan-area teenagers: 1971-1979. Fam Plam Perspect 1980; 12: 230-237.

27. Ringelin Y., Horwitz N., Maddaleno $\mathrm{M}$ et al. Un modelo psicosocial para el estudio de la adolescente. En: Temas de salud mental primaria en salud. Ed. Corporación Promoción Universitaria. 1991; 95-110.

28. Velasco FR. Los problemas del adolescente. Salud pública de México. 1984; 529-562.

29. Pérez J., Torres A. Repercusión del embarazo en salud perinatal de la adolescente. En: La psicología en el ámbito perinatal. INPER. México. 1988; 395-396.

30. Fernández A. Adolescentes embarazadas: relación de pareja y apoyos familiares. En: La psicología social en México. Asociación Mexicana de psicología social. 1988.

31. Molina R. Salud reproductiva del adolescente. Rev. Obstet. Ginecol. Venezuela 1992; 52(1): 3-7.

32. López LV., Sánchez J. Parto en la adolescente. Rev. Col. Obstet. Ginecol. 1993; 44(2): 133-137.

33. Esparza CA., Calle M. Características de las adolescentes obstétricas. Estudio comparativo con pacientes de 19-30 años. Hospital Universitario de Caldas. 1989. Rev. Col. Obstet. Ginecol. 1993; 44(2): 123 126.

34. Uriza G. Embarazo en adolescentes. Rev. Col. Obstet. Ginecol. 1983; 34(2): 102-114.

35. López G. Embarazo en adolescentes. Rev. Planificación, población y desarrollo. (Profamilia). 1992; 7(19): 31-32.

36. Monterrosa A. Incidencia de parto pretérmino y factores de riesgo. Rev. Col. Obstet. Ginecol. 1991; 42(3): 199-207.

37. Monterrosa A. Actitud obstétrica ante presentación podálica. Acta Médica de Cartagena. 1992; 3(2): 68-75.

38. Schwarz RL., Duverges CA., Gonzalo A., Fescina RH. Obstetricia. 4a. Ed. El Ateneo. Buenos Aires. 1986.

39. Rovinsky JJ. Anomalías de posición, situación, presentación y rotación. En: Iffy L. Kaminetz H.A. (Editores) Obstetricia y Perinatología. Principios y prácticas. Buenos Aires. Editorial Médica Panamericana. $1985 ; 927-944$

40. Kotagol U. Newborn consequences of teenager pregnancies. Pediatrics Annals. 1993; 22(2): 127-132.

41. Monterrosa A. Eclampsia en el Hospital de Maternidad Rafael Calvo. Rev. Col. Obstet. Ginecol. Ginecol. 1989; 40(3): 235-244.

42. Rebolledo A., Atalah E. Riesgos nutricionales en embarazadas adolescentes. Rev. Chil. Nutr. 1986; 14(3): 320-325.

43. Khwaja SS., Al-Sibai M., Al-Suleiman SA., Elizibedh MY. Obstetric implications of pregnancy in adolescence. Acta Obstet. Gynecol. Scand. 1986; 65: 57-61.

44. Slap GB., Schwartz J. Risk factor for low birth weight to adolescent mothers. J. Adolesc. Health Care. 1989; 10: 267-274.

45. Monterrosa A. Embarazo en adolescentes. El drama de las niñas madres. Rev Selecta Médica. 1994; 5(2): 7.

46. Stout J., Kiby D. The effects of sexuality educations on adolescent sexual activity. Pediatric Annals 1993; 22(2): 120-126.

47. Monterrosa A. Una educación para la sexualidad. Rev. Planificación, Población y Desarrollo (Profamilia). 1994; 11(23): 85-86. 